\title{
La contribución del entorno socioeconómico al autoconcepto y percepción infantil de las pautas parentales de crianza Gonzalo D. Clericil ${ }^{*}$, Ángel M. Elgier ${ }^{1,2,3}$, Lucas G. Gago-Galvagno 1,2,3, María Julia García ${ }^{1}$ y Susana C. Azzollini ${ }^{1,3}$
}

\author{
'Instituto de Investigaciones, Facultad de Psicología. Universidad de Buenos Aires. \\ ${ }^{2}$ Facultad de Psicología y Relaciones Humanas, Universidad Abierta Interamericana. \\ ${ }^{3}$ Consejo Nacional de Investigaciones Científicas y Técnicas, Argentina.
}

\begin{abstract}
Resumen: El objetivo general de la presente investigación ha sido analizar diferencias significativas en los modos en los que niños y niñas se perciben y autovaloran, y la percepción que poseen de las pautas de crianza de sus padres y madres, según el entorno socioeconómico. La muestra estuvo conformada con 105 niños y niñas de entre 7 y 12 años de edad, de la Ciudad Autónoma de Buenos Aires, Argentina. Para evaluar el autoconcepto se empleó la escala "Piers-Harris Children's Self Concept Scale. Second Edition" de Piers y Herzberg (2002). Para indagar la percepción infantil de las pautas parentales de crianza la versión española del P.E.P.P.E. ("Practiques Éducatives Parentales Perçues par l' Enfant") de Fortín, Cyr y Chénier (1997). Se encontró que los niños y niñas de entornos socioeconómicos más favorecidos presentaron niveles más elevados de autoconcepto y percepción de los estilos parentales de crianza con mayor presencia de apoyo parental. Se concluye que al ser los entornos socioeconómicos posibles mediadores de estas variables, es importante generar intervenciones tempranas y orientadas a generar mayores niveles de equidad social.
\end{abstract}

Palabras clave: Autoconcepto, Pautas parentales de crianza, Entorno socioeconómico, Niñez.

\section{The Contribution of the Socioeconomic Environment to the Child's Self-concept and Perception of Parental Rearing Styles}

\begin{abstract}
The general objective of this research has been to analyze significant differences in the ways in which boys and girls perceive and self-assess, and the perception they have of their parents' parenting guidelines, according to the socioeconomic environment. The sample was conform with 105 boys and girls between 7 and 12 years old, from the Autonomous City of Buenos Aires, Argentina. The Piers-Harris Children's Self Concept Scale (Second Edition, Piers \& Herzberg, 2002) was used to evaluate the selfconcept. To investigate the child's perception of parental parenting guidelines, the Spanish version of the P.E.P.P.E. ("Practiques Éducatives Parental Perçues par I' Enfant") of Fortín, Cyr and Chénier (1997). It was found that children from the most favored socio-economic environments presented higher levels of selfconcept and perception of parenting styles with a greater presence of parental support. It is concluded that since the socio-economic environments are possible mediators of these variables, it is important to implement early interventions aimed at generating higher levels of social equity.

Keywords: Self-concept; Parenting Guidelines; Socioeconomic Environment; Childhood.
\end{abstract}

Existen variadas definiciones acerca del autoconcepto como constructo teórico de larga producción en Psicología del Desarrollo. Thompson (1998) lo define como la construcción de lo que la persona percibe

Recibido: 27/08/2019 - Aceptado: 04/10/2019 - Avance online: 10/12/2019 *Correspondencia: Gonzalo Daniel Clerici.

Instituto de Investigaciones, Facultad de Psicología. Universidad de Buenos Aires.

C.P: C1052A, Buenos Aires, Argentina

E-mail: gclerici77@hotmail.com y valora de sí misma, y que se conforma en el marco de diversas experiencias socioculturales a lo largo de todo el ciclo vital. Piers y Herzberg (2002) por su parte, definen el autoconcepto como un conjunto relativamente estable de actitudes, que reflejan las descripciones y evaluaciones de la propia conducta y atributos que cada persona realiza de sí misma. Estos autores diferencian la dimensión conductual, desde la cual sostienen 
que es posible inferir la dimensión cognitiva del constructo. Por su parte, Purkey (1970) coincide en entender el autoconcepto como un producto de la interpretación y valoración que la persona realiza sobre sí misma, y profundiza el plano cognitivo al considerar la complejidad de la organización interna necesaria para procesar la información, guiar la conducta y acomodarse a las exigencias ambientales.

Las pautas parentales de crianza por su parte, suelen ser mencionadas también en términos de estilos parentales, estilos de educación familiar, o prácticas parentales de crianza. $Y$ se las define como los comportamientos y actitudes de los padres y madres en relación a sus hijos e hijas (Durning y Fortin, 2000). A su vez, las pautas parentales de crianza implican en líneas generales la combinación de dos dimensiones. Por un lado, el apoyo parental, definido como el grado en que los padres y madres intencionalmente alientan la autonomía, la autorregulación, la autoafirmación y la autovaloración por medio del apoyo y la sensibilidad, estando atentos a las necesidades y demandas de sus hijos e hijas; y por otro lado, el control parental, entendido como las demandas que padres y madres hacen a sus hijos e hijas para que estén integrados familiar y socialmente, incentivando su madurez, moldeando actitudes y comportamientos mediante monitoreos y supervisiones, brindando reglas y orientaciones, y manteniendo exigencias con el objetivo de lograr los criterios de adaptación social que esperan para ellos y ellas (Darling, 1999). La modalidad de interacción entre estas dos dimensiones de las pautas parentales de crianza ha dado a diversas tipologías de las dinámicas familiares. Los desarrollos de Baumrind (1967, 1971) han marcado un precedente en este sentido. Su tipología clásica ha sido sin duda la más influyente en la literatura. La misma distinguió tres tipos de crianza parental: "autoritaria" (modalidad que implica padres y madres exigentes y directivos, pero con niveles bajos de sensibilidad y empatía con las necesidades de sus hijos e hijas), "democrática" (padres y madres flexibles, exigentes y sensibles, cuyos métodos disciplinarios se orientan más hacia el apoyo que hacia la punición) y "permisiva" (padres y madres más sensibles que exigentes, poco coercitivos y nada severos, que habilitan conductas de llamativa autonomía en sus hijos e hijas, evitando la confrontación). Maccoby y Martin (1983) agregaron a esta tipología de Baumrind un cuarto tipo denominado "negligente", donde los niveles son bajos tanto en control como en apoyo o afecto parental. En este caso, padres y madres mostrarían altos niveles de indiferencia hacia sus hijos e hijas.

Por otro lado, en Argentina existe una percepción generalizada de que la educación pública ha ido perdiendo calidad con el tiempo, ya que los grupos menos carenciados han migrado de la escuela pública a la privada a lo largo de los años. Esto genera lo que se llama "segregación escolar" que conlleva un diferencial en los entornos socioeconómicos de los dos tipos de establecimiento educativo, siendo que en el público ingresan familias con menores niveles educativos y de ingresos (Adrogué y Orlicki, 2018; Gasparini, Jaume, Serio y Vázquez, 2011 ).

Estos entornos socioeconómicos se relacionan a una multiplicidad de variables. Es por esto que la vulnerabilidad social es considerada una problemática multidimensional, ya que se manifiestan de diversas maneras según el contexto sociocultural (Hauser y Labin, 2018). Según los informes de distintos organismos (CEPAL, 2018; Salvia y Bonfiglio, 2019) las personas que cumplen con los criterios de pobreza multidimensional en Argentina ya alcanzaron un tercio de la población general. Y el crecimiento se mantiene constante. Este tipo de entornos puede incluir privaciones de derechos, vinculados a aspectos económicos, educativos, ocupacionales, sociales y de vivienda (Brugué, Gomà y Subirats, 2018; Gutiérrez, 2018). Y traen aparejadas dificultades para el desarrollo de niños y niñas, tanto a nivel individual como familiar.

En este sentido el nivel educativo de padres y madres es la variable con mayor poder predictivo para el desarrollo infantil (Montroy et al., 2016; Neidhöfer, Serrano y Gasparini, 
2018). En estos entornos la cantidad de años que la persona pasa en espacios educativos disminuye significativamente. $Y$ esto influye en las expectativas con respecto a la educación de los hijos e hijas (Schoenfelder, Tein, Wolchik y Sandler, 2015) y en el tipo de interacciones y en la calidad de las mismas (Cadima, Verschueren y Guedes, 2016). En este sentido, la teoría ecológica del desarrollo de Bronfenbrenner (1987) sostiene que las familias y sus prácticas de crianza están incluidas ("anidadas") en una variedad de sistemas eco-sociales más abarcativos, que comprenden los sistemas de apoyo formal e informal, así como los valores socio-culturales de cada época.

Son varias las investigaciones que demuestran la relación que existe entre los diferentes entornos socioeconómicos y las pautas parentales de crianza. En algunas de ellas se comprobó que estas pautas son un mediador parcial del impacto del nivel socioeconómico sobre la salud infantil de edad preescolar y de los primeros años escolares (Belsky, Bell, Bradley y Stallard, 2006; Zilberstein, 2016). Sin embargo, a pesar de las condiciones socioeconómicas en las que se desarrollan niños y niñas, las pautas de crianza con niveles altos de apoyo parental son un factor protector de los efectos negativos que generan las situaciones de vulnerabilidad social, generando influencias en otras variables tales como la delincuencia, la salud mental y los logros educativos de niños y niñas (Kaiser, Pollmann-Schult y Song, 2017). El tipo de pauta de crianza que la familia utiliza para con sus hijos e hijas varía en función del contexto y la situación en la que viven, aunque los resultados no están exentos de contradicciones, ya que la relación entre ambas variables es compleja (Anton, Jones y Youngstrom, 2015; Roubinov y Boyce, 2017). En este sentido, Roubinov y Boyce (2017) encontraron que los entornos socioeconómicos se relacionaban con los estilos parentales de forma indirecta ya que ocasiona una variación en la salud mental parental, acceso a recursos y valores culturales. Es así como las pautas de crianza en entornos vulnerables traen aparejadas relaciones más punitivas, de obediencia y negligentes (Hoff,
Laursen y Tardif, 2002; Richaud et al., 2013), generan mayor nivel de estrés (Jones-Mason, Coccia, Grover, Epel y Bush, 2018) y mayor exposición a la violencia (Lipina y González, 2011; Richaud et al., 2013). Estos factores podrían ser tomados como mediadores entre ambas variables (Anton et al., 2015).

A su vez, los diversos entornos socioeconómicos también generarían un impacto en el autoconcepto de niños y niñas, en dimensiones tales como la autopercepción corporal, el rendimiento académico y la autopercepción familiar y social. Sin embargo, no es clara esta relación en la literatura científica (Easterbrook, Kuppens y Manstead, 2019; Gaeta y Cavazos, 2017; Tabernero, Serrano y Mérida, 2017). Yesto podría deberse al énfasis que cada sector socioeconómico les asigna a las diversas dimensiones que conforman el autoconcepto global (Destin, Rheinschmidt-Same y Richeson, 2017; Easterbrook et al., 2019). Por ende, además de que no son muchas las investigaciones que indagan esta asociación (Manstead, 2018; Nolasco, Osorio, Vázquez y Raya, 2017) los resultados son contradictorios, debido a que hay muchas variables intervinientes, tales como los altos niveles de resiliencia, las especificidades culturales de cada sector o el estilo de pautas de crianza, entre otros (Destin et al., 2017; Hincapié, Montoya y Dussan, 2016).

Como ya se subrayó, debido a la ampliación constante de los entornos vulnerables en la Argentina (CEPAL, 2018), a la escasez de estudios en esta temática (Manstead, 2018), a los resultados contradictorios encontrados debido a la complejidad de la temática (Destin et al., 2017; Roubinov y Boyce, 2017) y al diferencial socioeconómico que se observa en relación al ámbito educativo (público y privado) es que se vuelve necesario generar conocimiento que apunte no sólo a contribuir a la teoría, sino también a poder generar intervenciones con vistas a garantizar un desarrollo integral y saludable de niños y niñas. El objetivo de la siguiente investigación será analizar el impacto de los entornos socioeconómicos en el autoconcepto y la percepción infantil de las pautas parentales de crianza. Se espera encontrar un impacto 
de los entornos socioeconómicos tanto en las diferentes dimensiones del autoconcepto de los niños y niñas, como en su percepción de las pautas parentales de crianza de sus padres y madres, siendo que los entornos más favorecidos presentarán niveles más adecuados y elevados de estas variables.

\section{MÉTODO}

\section{DISEÑO}

El estudio aplicó un diseño cuantitativo, de alcance descriptivo, comparativo y de corte transversal.

\section{PARTICIPANTES}

La muestra de la presente investigación, de tipo no probabilística incidental, estuvo conformada por 105 díadas madre y padre con sus niños y niñas de entre 7 años y 0 meses, y 12 años y 11 meses de edad $(M=$ 9.58; $D E=1.71)$.

En cuanto al tipo de establecimiento educativo, el $50.5 \%$ de los niños y niñas concurrían a una escuela de gestión pública de la Ciudad Autónoma de Buenos Aires, Argentina, y el restante $49.5 \%$ a una escuela de gestión privada de la misma ciudad. La Tabla 1 resume los datos sociodemográficos con respecto a las variables socioeconómicas relativas a la muestra evaluada.
Se aplicó la prueba Chi-cuadrado para evaluar si existían diferencias significativas en los grupos en función de sus características. Con respecto al nivel educativo de los padres, se encontró una asociación estadísticamente significativa con el ámbito educativo $\left(X^{2}{ }_{(2)}=8.01 ; p=\right.$ $.018 ;$ V de Cramer $=.28)$. Lo mismo sucedió con la situación de empleo de los padres $\left(X^{2}{ }_{(1)}=38.05, p=.001 ; V\right.$ de Cramer $\left.=.62\right)$ y el recibir asistencia social $\left(X^{2}{ }_{(1)}=36.14\right.$; $p=.001 ; V$ de Cramer $=.61)$. En resumen, existen diferencias relevantes respecto al nivel socioeconómico de los padres y de las madres de los niños y niñas que asisten a la escuela de gestión pública, y de quienes asisten a la escuela de gestión privada. Estas diferencias se expresan en cuanto al nivel educativo de las madres, al porcentaje de personas adultas desempleadas pertenecientes a una y a otra escuela, como así también en cuanto al porcentaje de padres y madres que afirmaron recibir algún tipo de ayuda social. Es así como los datos socio-demográficos relevados evidencian la existencia de diferencias socioeconómicas entre los padres y madres de ambas escuelas, en aspectos tan centrales como el nivel educativo, el acceso al sistema laboral con todo lo que ello implica, y en el estar en situación de requerir de la ayuda social por parte del estado.

Tabla 1

Características sociodemográficas de la muestra

\begin{tabular}{|c|c|c|c|c|c|c|}
\hline \multirow{2}{*}{ Características } & \multicolumn{2}{|c|}{ Escuela Pública } & \multicolumn{2}{|c|}{ Escuela Privada } & \multicolumn{2}{|c|}{ Total } \\
\hline & $f$ & $\%$ & $f$ & $\%$ & $f$ & $\%$ \\
\hline Masculino & 27 & 25.7 & 26 & 24.8 & 53 & 52.4 \\
\hline Femenino & 26 & 24.8 & 26 & 24.7 & 52 & 47.6 \\
\hline Terciario/universitario & 11 & 10.5 & 23 & 22.0 & 34 & 32 \\
\hline Secundario & 23 & 22.0 & 20 & 19.0 & 43 & 41 \\
\hline Primario únicamente & 19 & 18.0 & 9 & 8.5 & 28 & 27 \\
\hline Empleado & 12 & 11.5 & 44 & 42.0 & 56 & 53.0 \\
\hline Desempleado & 41 & 39.0 & 8 & 7.5 & 49 & 47.0 \\
\hline No recibe asistencia & 14 & 13.5 & 45 & 43.0 & 59 & 56.0 \\
\hline Recibe asistencia & 39 & 37.0 & 7 & 6.5 & 46 & 44.0 \\
\hline Total & 53 & 50.5 & 52 & 49.5 & 105 & 100 \\
\hline
\end{tabular}




\section{INSTRUMENTOS DE MEDIDA}

\section{Cuestionario sociodemográfico}

Se administró un cuestionario sociodemográfico ad-hoc para recabar información sobre el entorno socioeconómico de los participantes. Se realizaron preguntas con respuesta cerrada sobre el nivel educativo alcanzado por los padres, si poseían empleo y si recibían ayudas económicas por parte del estado nacional.

\section{Autoconcepto infantil}

Se administró la escala "Piers-Harris Children's Self Concept Scale. Second Edition" desarrollada por Piers y Herzberg (2002) en Estados Unidos, a fin de indagar el autoconcepto de niños y niñas. Dicha escala se basa en una serie de supuestos acerca del autoconcepto: no es observable, por lo que debe inferirse a partir de autodescripciones; es una construcción global aunque con componentes específicos, dimensiones o dominios; es relativamente estable a lo largo del tiempo para cada quien; posee un componente descriptivo y uno evaluativo; sufre modificaciones a lo largo del desarrollo evolutivo; y cumple un papel importante en cuanto a la interacción con el medio, dado que incluye creencias, emociones y valores que determinan actitudes y motivaciones. Consta de seis sub-escalas a fin de abordar las diferentes dimensiones del autoconcepto: Conducta; Estatus intelectual y escolar; Apariencia y atributos físicos; Ansiedad; Popularidad; y Felicidad y satisfacción.

Esta versión de la escala no ha sido aún validada ni instrumentada en Argentina, aunque sí existe una versión validada pero anterior (1984), implementada por Casullo (1990). Es a su vez, una escala que, en sus diversas versiones, ha sido administrada en diferentes países latinoamericanos. La versión de 2002 cuenta con 60 ítems que se contestan por Si o por No. El puntaje total puede variar entre 0 y 72 puntos (ya que cada ítem puede formar parte de más de una subescala) y refleja el número de ítems que han sido contestados en la dirección del autoconcepto positivo en las diferentes subescalas. De manera que un puntaje alto indica un autoconcepto global positivo, mientras que los puntajes bajos se asocian a un autoconcepto global negativo. Se adjudica 1 punto por cada respuesta en la dirección del autoconcepto positivo. La utilización de las sub-escalas posibilita generar hipótesis acerca de las áreas de fortaleza o vulnerabilidad del niño o de la niña. Esta adaptación de la escala puede administrarse a niños y niñas de entre 7 y 12 años de edad. Se determinó la fiabilidad de la escala a través con la fórmula 20 de Kuder-Richardson, la cual arrojó un índice de consistencia interna de 0.89 .

Percepción infantil de las pautas parentales de crianza

Se instrumentó la adaptación castellana del P.E.P.P.E. ("Practiques Éducatives Parentales Perçues par I'Enfant") de Fortín, Cyr y Chénier (1997), adaptación a su vez del P.P.I. ("Parental Perception Inventory") de Hazzard, Christensen y Margolin (1983). La misma es una escala destinada a evaluar la percepción que niños y niñas tienen de las prácticas de crianza parentales de las que participan. Es aplicable a niños y niñas de entre 5 a 13 años de edad. Se presenta como un cuestionario en el curso del cual el niño o la niña deben evaluar a cada uno de sus progenitores a partir de la descripción de ciertos comportamientos. Estos comportamientos se agrupan en 18 ítems, 9 para la dimensión apoyo parental y 9 para la dimensión control. Asu vez, la dimensión apoyo parental incluye 9 indicadores: recompensar ante actitudes o comportamientos evaluados positivamente; reconfortar emocionalmente ante situaciones adversas; dedicar tiempo de conversación; alentar la participación en la toma de decisiones; generar espacios compartidos; elogiar, realizar evaluaciones positivas; favorecer la autonomía; asistir ante las dificultades de los niños; y realizar demostraciones de afecto no verbal. Por su parte, la dimensión control parental incluye 9 indicadores (cada una de ellas representada por un ítem): remover privilegios 
ante comportamientos no deseables; realizar críticas ante conductas evaluadas como negativas 0 incorrectas; ordenar el funcionamiento de los niños; castigar físicamente; gritar; amenazar; sancionar con penitencias; insistir con señalamientos de modo perseverante; e ignorar como modo de reprobación de actitudes y conductas. Para cada uno de los ítems, tanto para los referidos al apoyo, como los referidos al control, se evalúa la frecuencia del comportamiento parental en base a una escala Likert de 5 puntos $(0=$ nunca; $4=$ siempre $)$, tanto para la madre, como para el padre. El puntaje total de los comportamientos referidos al apoyo y al control en cada caso, puede variar entre 0 y 36 puntos. En el presente estudio la consistencia interna ( $\alpha$ de Cronbach) de la subescala de apoyo parental fue de 0.86 y la de control fue de 0.88 .

\section{PROCEDIMIENTO}

Se seleccionó una escuela de gestión pública y una escuela de gestión privada de la Ciudad Autónoma de Buenos Aires. Se realizaron entrevistas con las autoridades de las mismas para informarles sobre el proyecto de trabajo y las edades de los niños y niñas que serían seleccionados para la muestra. Se realizó el trabajo de campo con los niños y niñas luego de haber obtenido el correspondiente consentimiento por parte de sus padres y madres, a quienes se les explicaron los fines de la investigación y se les explicitó que la misma era anónima y confidencial. La investigación contó, a su vez, con el aval del Ministerio de Educación del Gobierno de la Ciudad Autónoma de Buenos Aires.

Todas las pruebas fueron entregadas en el mismo orden (Autoconcepto infantil y Pautas parentales percibidas) y por el mismo evaluador masculino, para realizar control por equiparación. Los instrumentos fueron aplicados de modo individual con cada niño y niña, en un aula silenciosa, con la iluminaria adecuada, y apartada de cada establecimiento educativo.

\section{ANÁLISIS DE DATOS}

Para el procesamiento estadístico de la información cuantitativa se generaron bases de datos específicas con el paquete estadístico IBM SPSS Statistics en su versión

Tabla 2

Diferencia de medias de autoconcepto global y de cada dimensión según tipo de establecimiento educativo de los niños encuestados (Prueba t de Student)

\begin{tabular}{|c|c|c|c|c|c|c|c|}
\hline & $\begin{array}{l}\text { Tipo de } \\
\text { escuela }\end{array}$ & $n$ & $M$ & $D E$ & $t$ & $p$ & $\mu^{2}$ \\
\hline \multirow{2}{*}{ Autoconcepto global } & Pública & 53 & 49.30 & 13.36 & $-3.87 * \star$ & .001 & .126 \\
\hline & Privada & 52 & 57.90 & 9.02 & & & \\
\hline \multirow{2}{*}{ Conducta } & Pública & 53 & 10.21 & 2.82 & $-3.56^{\star \star}$ & .001 & .109 \\
\hline & Privada & 52 & 11.92 & 2.07 & & & \\
\hline \multirow{2}{*}{ Intelectualidad } & Pública & 53 & 10.34 & 3.12 & $-2.38^{*}$ & .019 & .052 \\
\hline & Privada & 52 & 11.63 & 2.41 & & & \\
\hline \multirow{2}{*}{ Apariencia } & Pública & 53 & 6.96 & 2.79 & -1.78 & .078 & .030 \\
\hline & Privada & 52 & 7.83 & 2.16 & & & \\
\hline \multirow{2}{*}{ Ansiedad } & Pública & 53 & 7.66 & 3.82 & $-2.62^{\star \star}$ & .010 & .062 \\
\hline & Privada & 52 & 9.33 & 2.61 & & & \\
\hline \multirow{2}{*}{ Popularidad } & Pública & 53 & 6.81 & 2.82 & $-5.04 * \star$ & .001 & .197 \\
\hline & Privada & 52 & 9.31 & 2.22 & & & \\
\hline \multirow{2}{*}{ Felicidad } & Pública & 53 & 7.32 & 1.65 & -1.97 & .051 & .036 \\
\hline & Privada & 52 & 7.88 & 1.25 & & & \\
\hline
\end{tabular}


24. Posteriormente, se realizó un análisis descriptivo de cada una de las variables que componen este estudio, con la prueba Chicuadrado para encontrar diferencias entre las características de los grupos, y finalmente se emplearon pruebas de comparación de medias (Prueba $t$ de Student).

\section{RESULTADOS}

En lo que refiere al autoconcepto global, se observa una diferencia estadísticamente significativa entre las medias de los niños y las niñas que asisten a la escuela de gestión pública y de quienes asisten a la escuela de gestión privada. Por lo tanto, de acuerdo a los resultados hallados, podría afirmarse que los niños y niñas que asisten a escuela privada presentan un mayor autoconcepto global positivo en comparación con aquellos que asisten a la escuela pública.

En cuanto a las diferencias en las distintas dimensiones del autoconcepto, se observan diferencias en las dimensiones: Conducta, Intelectualidad, Ansiedad y Popularidad. En todos los casos, los niños y niñas que asisten a la escuela de gestión privada evidencian medias superiores, por lo que podría afirmarse que se autoperciben más disciplinados, más satisfechos con su rendimiento escolar, menos ansiosos y más populares en comparación con quienes asisten a la escuela de gestión pública. La Tabla 2 resume los datos descriptivos y comparativos.

En cuanto a la percepción infantil del apoyo materno, los niños y las niñas que asisten a la escuela privada perciben un mayor apoyo por parte de sus madres.

Respecto al control materno, si bien no alcanza la significación estadística, el bajo nivel de error $(p=.074)$, permitiría suponer una tendencia que indicaría que los niños y niñas que concurren a la escuela pública percibirían un mayor control materno en comparación con los que asisten a la escuela privada. Sin embargo, cabe destacar que la variable tipo de escuela sólo explica un $6,1 \%$ de la varianza del apoyo materno y un $3,1 \%$ de la varianza del control materno.

En lo que refiere a la percepción infantil del apoyo por parte del padre, los niños y niñas de la escuela privada perciben un mayor apoyo paterno, en relación con quienes concurren a la escuela pública.

Por último, los niños y niñas que concurren a la escuela de gestión pública perciben un mayor control paterno, en comparación con los que asisten a la escuela privada. No obstante, la variable tipo de escuela explica

Tabla 3

Diferencia de medias de apoyo y de control parental percibido según tipo de establecimiento educativo de los niños encuestados (Prueba t de Student)

\begin{tabular}{|c|c|c|c|c|c|c|c|}
\hline & $\begin{array}{l}\text { Tipo de } \\
\text { escuela }\end{array}$ & $n$ & M & $D E$ & $t$ & $p$ & $\mu^{2}$ \\
\hline \multirow{2}{*}{$\begin{array}{c}\text { Apoyo } \\
\text { percibido por } \\
\text { parte de la } \\
\text { madre }\end{array}$} & Pública & 53 & 25.89 & 6.42 & $-2.61^{*}$ & .011 & .061 \\
\hline & Privada & 52 & 28.60 & 3.97 & & & \\
\hline \multirow{2}{*}{$\begin{array}{c}\text { Control } \\
\text { percibido por } \\
\text { parte de la } \\
\text { madre }\end{array}$} & Pública & 53 & 13.64 & 6.76 & 1.81 & .074 & .031 \\
\hline & Privada & 52 & 11.42 & 5.79 & & & \\
\hline \multirow{2}{*}{$\begin{array}{c}\text { Apoyo } \\
\text { percibido } \\
\text { por parte del } \\
\text { padre }\end{array}$} & Pública & 47 & 25.60 & 6.42 & $-2.47^{\star}$ & .015 & .062 \\
\hline & Privada & 47 & 28.66 & 5.57 & & & \\
\hline \multirow{2}{*}{$\begin{array}{c}\text { Control } \\
\text { percibido } \\
\text { por parte del } \\
\text { padre }\end{array}$} & Pública & 47 & 12.43 & 7.13 & $3.23 * \star$ & .002 & .102 \\
\hline & Privada & 47 & 8.34 & 4.94 & & & \\
\hline
\end{tabular}


un $6.2 \%$ de la varianza del apoyo paterno y un $10.2 \%$ de la varianza del control paterno. La tabla 3 resume los datos descriptivos y comparativos.

\section{DISCUSIÓN}

El objetivo de la siguiente investigación fue evaluar si existían diferencias entre el autoconcepto y la percepción de las pautas parentales de crianza en niños y niñas de un centro público y privado, con diversidad de nivel socioeconómico en cada uno de ellos. $\mathrm{Se}$ encontraron diferencias significativas, tanto en el autoconcepto de los niños y niñas, como así también en la percepción de las pautas parentales de crianza, al considerar el tipo de establecimiento escolar en el que se desarrollan.

Los niños y niñas que provienen de escuelas privadas, y por ende provenientes de entornos socioeconómicos más favorables, presentaron mayores niveles de autoconcepto global positivo, en comparación con aquellos niños y niñas provenientes de escuelas públicas. En este sentido, en 4 de las 6 dimensiones que componen el autoconcepto global se encontraron diferencias significativas (tanto en Conducta, como en Intelectualidad, Ansiedad y en Popularidad). Estos resultados coinciden con los hallados en estudios similares que sostienen que debe considerarse al estrés como un aspecto significativo de los entornos más desfavorecidos, y como un factor que incide negativamente sobre el desarrollo integral y saludable de niños y niñas (JonesMason et al., 2018; Richaud de Minzi et al., 2013).

A su vez, tal como es esperable en función de las relaciones halladas entre la percepción de las pautas parentales de crianza y el autoconcepto, se encontraron diferencias significativas al considerar la percepción infantil de las pautas parentales de crianza y el tipo de establecimiento educativo. Los niños y niñas que asisten a la escuela pública presentaron una media inferior de percepción de apoyo materno, que quienes asisten a la escuela de gestión privada. Inversamente, respecto a la percepción de control materno, la diferencia entre los valores permitiría suponer una tendencia que indicaría que quienes asisten a la escuela pública perciben un mayor control materno, en comparación con los que asisten a la escuela privada. Por su parte, en cuanto a la percepción de apoyo paterno, los niños y niñas que asisten a la escuela pública presentaron una media menor que los que asisten a la escuela privada. Y, por el contrario, quienes asisten a la escuela pública percibieron un mayor control paterno, en comparación con los que asisten a la escuela privada.

Estos datos coinciden con lo presentado por el Ministerio de Desarrollo Social y UNICEF Argentina (2013), donde se sostiene que, en el marco de una investigación realizada a nivel nacional, con una muestra de 25.400 hogares, encontraron que la aceptación del castigo físico como pauta parental de crianza, disminuye a medida que aumenta la cantidad de años promedio de educación formal de los padres y madres. Y a su vez, en los hogares donde las personas adultas cuentan con niveles educativos más altos, el castigo es desestimado como modalidad a implementar en la crianza. En este sentido, y considerando los resultados encontrados en la presente investigación, resulta importante diseñar proyectos de intervención centrados en los procesos de socialización familiar, especialmente destinados a aquellas familias que se encuentren insertos en escuelas públicas.

Asimismo, Alonso y Román (2003) en diferentes estudios realizados en España sostienen queen padresymadrespertenecientes a entornos socio-culturales vulnerables, la exigencia y el control priman por sobre la expresión del afecto en las pautas parentales de crianza, no favoreciendo el intercambio con los hijos e hijas, ni su autonomía. Por su parte, también Delucca y González (2010) han encontrado en nuestro país diferencias a nivel socio-cultural en las prácticas familiares. Diversos estudios sostienen que el estrés asociado a las situaciones de vulnerabilidad social se asocia al uso de estrategias de control parental. Sin embargo, no es tan sólo el incremento de actitudes y comportamientos 
poco deseables del control parental lo que suele asociarse a padres y madres en situación de vulnerabilidad social. Richaud et al. (2013) en una investigación realizada con niños y niñas en situación de vulnerabilidad social en Argentina y en España, encontraron diferencias culturales en las pautas parentales de crianza, presentando los padres y madres de Argentina niveles más altos de negligencia.

En cuanto a las limitaciones que presenta la investigación realizada, se pueden mencionar los siguientes: el tamaño de la muestra y el recorte geográfico no permiten generalizar los resultados obtenidos; el tipo de estudio, que si bien permite observar la existencia de relaciones significativas entre las variables, no permite establecer relaciones de causalidad/ explicativas; y que los instrumentos de medición utilizados no han sido validados en Argentina hasta el presente. Hay que subrayar además que los tamaños del efecto encontrados en las comparaciones de grupo fueron en general bajos, y que las diferencias encontradas según el ámbito educativo podrían deberse a otras variables no analizadas.

No obstante estas limitaciones, el estudio del autoconcepto y de la percepción infantil de las pautas parentales de crianza en niños y niñas en edad escolar resulta relevante, puesto que no se han hallado investigaciones sobre la relación entre estos constructos en nuestro contexto. Esta investigación es la primera en aportar resultados sobre cómo diferentes características del entorno en colegios de diferentes ámbitos educativos contribuyen a la forma en que niños y niñas perciben las pautas parentales de crianza y su propio autoconcepto en Buenos Aires, Argentina. La mayoría de las investigaciones han trabajado con reportes parentales de los estilos de crianza y con indicadores culturales en sectores vulnerables directamente, y no en diferencias socioeconómicas derivadas del ámbito educativo en Argentina. A su vez, refuerza los resultados encontrados en otras investigaciones provenientes de otros países.

Finalmente, este estudio puede contribuir a aportar evidencias a ser utilizadas en el diseño e implementación de proyectos de prevención del maltrato infantil mediante talleres grupales en colegios de sectores públicos. Asimismo, estos estudios pueden aportar datos para la formulación de programas de orientación a padres y madres sobre estilos y pautas de crianza, especialmente en entornos socialmente vulnerables. Y por último, pueden aportar evidencia para trabajar en el aula con el autoconcepto de los niños y niñas, para generar niveles más elevados del mismo y mejorar el clima escolar.

\section{- Conflicto de intereses.}

Los autores declaran no tener ningún conflicto de intereses.

\section{REFERENCIAS}

Adrogué, C., \& Orlicki, M.E. (2018). Evolución y perspectivas de la educación en Argentina, su cobertura y su calidad. Cultura Económica, 32(87), 1-15.

Alonso, J., \& Román, J. (2003). Educación familiar y autoconcepto en niños pequeños. Madrid: Editorial Pirámide.

Anton, M.T., Jones, D.J., \& Youngstrom, E.A. (2015). Socioeconomic status, parenting, and externalizing problems in African American single-mother homes: A person-oriented approach. Journal of Family Psychology, 29, 405. doi:10.1037/fam0000086

Baumrind, D. (1967). Childcare practices anteceding three patterns of preschool behavior. Genetic Psychology Monographs, 75 (2), 43-88.

Baumrind, D. (1971). Current patterns of parental authority. Developmental Psychology Monograph, 4, 1-14

Belsky, J., Bell, B., Bradley, R.H., \& Stallard, N. (2006). Socioeconomic risk, parenting during the preschool years. European Journal of Public Health, 17(4), 508-513.

Bronfenbrenner, U. (1987). La ecología del desarrollo humano. Barcelona: Paidós.

Brugué, Q., Gomà, R., \& Subirats, J. (2018). De la pobreza a la exclusión social. Nuevos retos para las políticas públicas. Revista Internacional de Sociología, 60(33), 7-45. doi:10.3989/ris.2002. i33.728

Cadima, J., Verschueren, K., Leal, T., 
\& Guedes, C. (2016). Classroom interactions, dyadic teacher-child relationships, and self-regulation in socially disadvantaged young children. Journal of Abnormal Child Psychology, 44(2), 7-17. doi: 10.1007/s10802015-0060-5

Casullo, M.M. (1990). El autoconcepto. Técnicas de evaluación. Buenos Aires: Psicoteca Editorial

CEPAL, N. (2018). Informe de actividades del Instituto Latinoamericano y del Caribe de Planificación Económica y Social (ILPES), 2017-2018. CEPAL.

Darling, N. (1999). Parenting style and its correlates. Washington: ERIC Digest

Delucca, N.E., González, M., \& Martínez, A. (2010). Modalidades de la diversidad en los vínculos familiares. Revista de Psicología-Segunda Época, 17 (2), 1-17.

Destin, M., Rheinschmidt-Same, M., \& Richeson, J.A. (2017). Statusbased identity: A conceptual approach integrating the social psychological study of socioeconomic status and identity. Perspectives on Psychological Science, 12(3), 270-289. doi:10.1177/1745691616664424

Durning, P., \& Fortin, A. (2000). Les pratiques éducatives parentales vues par les enfants. Enfance, 4(3), 375-391.

Easterbrook, M.J., Kuppens, T., \& Manstead, A.S. (2019). Socioeconomic status and the structure of the self-concept. British Journal of Social Psychology, 27(1), 17 29. doi:10.1111/biso.12334

Fortin, A., Cyr, M., \& Chénier, N. (1997). Inventaire du comportement parental vu par l'enfant (trad. francesa de Hazzard, A., Christensen, A. y Margolin, G., Parental perception inventory). Montreal, Canada: Université de Montréal.

Gaeta, M.L., \& Cavazos, J. (2017). Autoconcepto físico y académico en niños de contextos marginados en México. Revista electrónica de investigación educativa, 19(2), 114-124. doi:10.24320/redie.2017.19.2.604

Gasparini, L., Jaume, D., Serio, M., \& Vázquez, E. (2011). La segregación entre escuelas públicas y privadas en Argentina. Reconstruyendo la evidencia. Desarrollo Económico: Revista de Ciencias Sociales, 34(2), 189-219.

Gutiérrez, E.M. (2018). Vulnerabilidad y exclusión social en Argentina: un análisis durante el período 2006-2016. Sudamérica: Revista de Ciencias Sociales, $17(9), 188-218$.

Hauser, M.P., \& Labin, A. (2018). Evaluación cognitiva de niños: un estudio comparativo en San Luis, Argentina. Revista Costarricense de Psicología, 37(2), 27-40. doi:10.22544/rcps. v37i01.02

Hazzard, A., Christensen, A., \& Margolin, G. (1983): Children's perceptions of parental behaviors. Journal of Abnormal Child Psychology, 17, 49-60.

Hincapié, L.M., Montoya, D.M., \& Dussan, C. (2016). Caracterización del autoconcepto en niños y niñas en situación de desplazamiento en Mocoa, Colombia. Revista de Investigaciones UCM, 16(3), 118-132. doi:10.22383/ ri.v16i1.64

Hoff, E., Laursen, B., Tardif, T., \& Bornstein, M. (2002). Socioeconomic status and parenting. Handbook of parenting Volume 2: Biology and ecology of parenting, 8, 231-52.

Jones-Mason, K.M., Coccia, M., Grover, S., Epel, E.S., \& Bush, N.R. (2018). Basal and reactivity levels of cortisol in onemonth-old infants born to overweight or obese mothers from an ethnically and racially diverse, low-income community sample. Psychoneuroendocrinology, 88(1), 115-120. doi:10.1016/i. psyneven.2017.12.001

Kaiser, T., Li, J., Pollmann-Schult, M., \& Song, A. (2017). Poverty and child behavioral problems: the mediating role of parenting and parental well-being. International journal of environmental research and public health, 14(2), 981. doi:10.3390/ijerph 14090981

Lipina, S.J., \& González, M.Á.Á. (2011). Contribuciones de la neurociencia cognitiva al diseño de políticas científicas 
y sociales para niños en situación de pobreza. Interamerican Journal of Psychology, 45(2), 243-253.

Maccoby, E.E., \& Martin, J.A. (1983). Socialization in the context of the family: Parent-child interaction. En P.H. Mussen (Ed.), Handbook of Child Psychology (pp. 1-101). Nueva York: J. Wiley.

Manstead, A.S. (2018). The psychology of social class: How socioeconomic status impacts thought, feelings, and behaviour. British Journal of Social Psychology, 57(2), 267-291. doi:10.1111/biso.12251

Ministerio de Desarrollo Social - UNICEF Argentina (2013). Encuesta sobre Condiciones de Vida de Niñez y Adolescencia. Principales Resultados. Buenos Aires: Ministerio de Desarrollo Social, Presidencia de la Nación UNICEF Argentina.

Montroy, J.J., Bowles, R.P., Skibbe, L.E., McClelland, M.M., \& Morrison, F.J. (2016). The development of selfregulation across early childhood. Developmental Psychology, 52(11), 1744. doi:10.1037/dev0000159

Neidhöfer, G., Serrano, J., \& Gasparini, L. (2018). Educational inequality and intergenerational mobility in Latin America: A new database. Journal of Development Economics, 134, 329-349. doi:10.1016/i.ideveco.2018.05.016

Nolasco, E.C., Osorio, K.A.C., Vázquez, M.C.P., \& Raya, E.J.V. (2017). Influencia del estado socioeconómico y civil de los padres en el autoconcepto de niños. PsicoEducativa: reflexiones y propuestas, 3(5), 24-30.

Piers, E.V., \& Herzberg, D.S. (2002). PiersHarris Children's Self Concept Scale, Second Edition. Los Ángeles, CA: W. P. S.

Purkey, W.W. (1970). Self-concept and school achievement. Englewood Cliffs, NJ: Prentice Hall.

Richaud, M.C., Mesurado, B., SamperGarcía, P., Llorca, A., Lemos, V., \& Tur, A. (2013). Estilos parentales, inestabilidad emocional y agresividad en niños de nivel socioeconómico bajo en Argentina y España. Ansiedad y estrés, 19(1), 5369.

Roubinov, D.S., \& Boyce, W.T. (2017). Parenting and SES: relative values or enduring principles? Current opinion in Psychology, 15, 162-167. doi:10.1016/i. copsyc. 2017.03 .001

Salvia, A., \& Bonfiglio, J.I. (2019). Pobreza multidimensional fundada en derechos económicos y sociales: Argentina urbana: 2010-2018. Observatorio de la Deuda Social Argentina. Universidad Católica Argentina.

Schoenfelder, E.N., Tein, J.Y., Wolchik, S., \& Sandler, I.N. (2015). Effects of the family bereavement program on academic outcomes, educational expectations and job aspirations 6 years later: The meadiating role of parenting and youth mental health problems. Journal of abnormal child psychology, 43(2), 229 241.

Tabernero, C., Serrano, A., \& Mérida, R. (2017). Estudio comparativo de la autoestima en escolares de diferente nivel socioeconómico. Psicología Educativa, 23(1), 9-17. doi:10.1016/i. pse.2017.02.001

Thompson, R.A. (1998). Early sociopersonality development. En W. Damon \& N. Eisenberg (Eds.), Handbook of child psychology: Social, emotional, and personality development (pp. 25104). Hoboken, NJ: John Wiley \& Sons Inc.

Zilberstein, K. (2016). Parenting in families of low socioeconomic status: A review with implications for child welfare practice. Family court review, 54(2), 221 231. doi: $10.1111 /$ fcre. 12222 